\title{
LORENZ HALBEISEN
}

\section{NORBERT HUNGERBÜHLER}

\section{The Josephus problem}

Journal de Théorie des Nombres de Bordeaux, tome 9, no 2 (1997), p. 303-318

<http://www.numdam.org/item?id=JTNB_1997_9_2_303_0>

(C) Université Bordeaux 1, 1997, tous droits réservés.

L'accès aux archives de la revue «Journal de Théorie des Nombres de Bordeaux » (http://jtnb.cedram.org/) implique l'accord avec les conditions générales d'utilisation (http://www.numdam.org/conditions). Toute utilisation commerciale ou impression systématique est constitutive d'une infraction pénale. Toute copie ou impression de ce fichier doit contenir la présente mention de copyright.

\section{Numdam}

Article numérisé dans le cadre du programme

Numérisation de documents anciens mathématiques

http://www.numdam.org/ 
Journal de Théorie des Nombres

de Bordeaux 9 (1997), 303-318

\title{
The Josephus Problem
}

\author{
par LORENZ HALBEISEN ET NORBERT HUNGERBÜHLER
}

\begin{abstract}
RÉSumÉ. Nous donnons des formules explicites permettant de calculer les nombres de Josephus $j(n, 2, i)$ and $j(n, 3, i)$ et fournissant une majoration et une minoration explicites de $j(n, k, i)$ qui ne diffèrent que d'au plus $2 k-2$ (dans le cas $k=4$, ces bornes sont même meilleures). Nous proposons aussi un nouvel algorithme pour le calcul de ces nombres basé précisément sur ces estimations.
\end{abstract}

Abstract. We give explicit non-recursive formulas to compute the Josephus-numbers $j(n, 2, i)$ and $j(n, 3, i)$ and explicit upper and lower bounds for $j(n, k, i)$ (where $k \geq 4$ ) which differ by $2 k-2$ (for $k=4$ the bounds are even better). Furthermore we present a new fast algorithm to calculate $j(n, k, i)$ which is based upon the mentioned bounds.

\section{Introduction}

The Josephus problem in its original form goes back to the Roman historian Flavius Josephus (see [3]). In the Romano-Jewish conflict of $67 \mathrm{~A}$. D., the Romans took the town Jotapata which Josephus was commanding. He and 40 companions escaped and were trapped in a cave. Fearing capture they decided to kill themselves. Josephus and a friend did not agree with that proposal but were afraid to be open in their opposition. So they suggested that they should arrange them in a circle and that counting around the circle in the same sense all the time, every third man should be killed until there was only one survivor who would kill himself. By choosing the position 31 and 16 in the circle, Josephus and his companion saved their lives. (How Josephus became Roman historian is another interesting story.)

Let us fix some notations in order to describe this problem generally. We number the $n$ positions in the circle by $0,1,2, \ldots, n-1$ and start counting 
at number 0 . Then every $k$ th element is removed. We define

$$
j(n, k, i), \quad(n \geq 1, k \geq 1,1 \leq i \leq n),
$$

to be the number of the $i$ th element which is removed by the process described above (see the example in Figure 1).
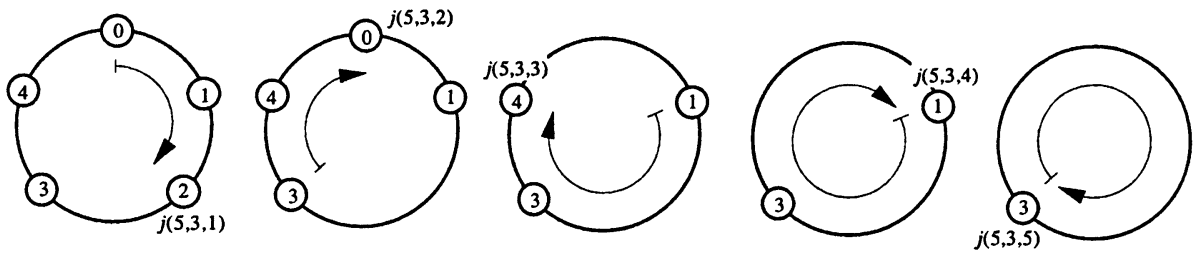

FIGURE 1. The Josephus sequence for $n=5, k=3$.

Numerous aspects of the Josephus problem and properties of the function $j$ are treated in the literature: In [5] the structure of the permutation

$$
\left(\begin{array}{ccccc}
0 & 1 & 2 & \ldots & n-1 \\
j(n, k, 1) & j(n, k, 2) & j(n, k, 3) & \cdots & j(n, k, n)
\end{array}\right)
$$

is investigated. In [7] a recursion formula for $j(n, k, n)$ is derived (there is some difference to our notation) and also some congruence properties of the Josephus numbers. In [2] a recursive algorithm is given to calculate the function $j$ and to solve the equation $j(n, k, i)=l$ for $i$ when $n$ and $k$ are given. In [1] an interpretation of the Josephus problem is given in terms of the representation of rational numbers over the rational base $\frac{k}{k-1}$. Note that the rational number $\frac{k}{k-1}$ plays an important role also in this work.

However, no explicit formula for the function $j$ is known. It is the aim of this article to give explicit (non-recursive) upper and lower bounds for the value of $j(n, k, i)$. These bounds coincide in case $k=2, k=3$ (and hence this yields an explicit formula in the mentioned cases) and in the case $k=4$ at least the so called collapsing numbers are determined exactly such that the resulting formula is exact in most cases (however it may happen, that upper and lower bound differ by 1 ). For $k \geq 5$ the upper and the lower bound differ by $2 k-2$ such that at least for circles larger than $n=2 k-2$ we can say who is not the $i$ th element to be removed.

In Section 6 we also present a new algorithm to compute $j(n, k, l)$ for general $n, k, l$ which is based upon the formulas for the mentioned bounds.

To finish this introduction we want to clarify what we mean by "explicit formula": 
Definition We define the set of explicit functions $f: \mathbb{C} \rightarrow \mathbb{C}$ (or $f: \mathbb{R} \rightarrow$ R) as follows:

- $f$ is explicit if $f$ is a constant (complex) function or the identity,

- if $f$ is explicit then $F(f)$ is explicit if $F$ is one of the functions exp, $\log$ (the principal branch and $\log (0):=0$ ) or (if $f$ is a real function) floor,

- if $f$ and $g$ are explicit then $f \diamond g$ is explicit if $\diamond$ is one of the binary functions,,$+- *$ (multiplication), / (division, and $\frac{c}{0}:=0$ for $c \in$ $\mathbb{C})$, ○ (composition).

A function $f\left(n_{1}, \ldots, n_{k}\right)$ is said to be explicit in $n_{i}$ if for any fixed $n_{j}$, $j \neq i$, the function $n_{i} \mapsto f\left(n_{1}, \ldots, n_{k}\right)$ is explicit.

This definition suffices for our purposes since it contains also constructions like $f(n)=\max (g(n), h(n)), f(n)=\operatorname{Round}(g(n))$ or

$$
f(n)= \begin{cases}g(n) & \text { if } h(n)>k(n) \\ l(n) & \text { otherwise }\end{cases}
$$

as one easily can see. But in general a larger class is used to treat other types of difference equations (see e.g. [4]).

An example is the well-known explicit formula

$$
f(n)=\operatorname{Round}\left(a \lambda^{n}\right)
$$

with

$$
\lambda=\lim _{j \rightarrow \infty} \frac{f(j+1)}{f(j)}, \quad a=\frac{2+\lambda}{5}
$$

for the Fibonacci numbers which are recursively given by $f(0)=f(1)=1$, $f(n+2)=f(n+1)+f(n)$. Of course, for concrete calculations one has to compute an approximation for the number $\lambda$ either by $\lambda \approx \frac{f\left(j_{0}+1\right)}{f\left(j_{0}\right)}$ for some large $j_{0}$ or by an algorithm which provides numerical approximations of $\sqrt{5}$ ( $j$ for which one wants to know $f(j)$.

\section{A recursive formula for $j(n, k, i)$}

A special case of the following formula may be found e.g. in [7]. However we will give a much simpler proof. 
THEOREM 1. For the josephus function $j(n, k, i)$ the following recursion holds

(1)

$$
j(n+1, k, i+1)=(k+j(n, k, i)) \bmod (n+1), \quad(n \geq 1, k \geq 1,1 \leq i \leq n)
$$

with initial value

$$
j(n, k, 1)=(k-1) \bmod n, \quad(n \geq 1, k \geq 1) .
$$

Remark: By " $a \bmod b$ " we mean the non-negative integer remainder of the division of $a$ by $b$.

Proof: The formula (2) follows directly from the definition. To see (1) we proceed by induction. Suppose, we know the value of $j(n, k, i)=: g$. Hence, if we start counting at number 0 , the $i$ th member removed is number $g$. Now consider $j(n+1, k, i+1)$. Because of $j(n+1, k, 1)=(k-1) \bmod (n+1)$, in the first step number $(k-1) \bmod (n+1)$ is removed and for the second step we start counting at number $k$. Therefore the problem is to find the $i$ th member which is removed (after removing $(k-1) \bmod (n+1))$ when we start counting at number $k$. But this is $(g+k) \bmod (n+1)$. So, we get (1).

\section{A recursive formula for the collapsing numbers $c_{m}$}

Consider for fixed $k>1$ and $l \geq 0$ and for variable $n>l$ the recursive formula $j(n+1, k, n+1-l)=(k+j(n, k, n-l)) \bmod (n+1)$ with $j(n, k, 1)=$ $(k-1) \bmod n$.

Definition. If $j(n, k, n-l) \leq k-2$ we call $n$ a collapsing number, otherwise we call $n$ regular.

The reason for this terminology is that regular numbers $n$ are characterized by the property that

$$
j(n, k, n-l)=j(n-1, k, n-l-1)+k
$$

if the right hand side exists. We claim that for the first collapsing number $c_{1}$ there holds

$$
c_{1}=l+\left\lceil\frac{l+1}{k-1}\right\rceil,
$$

where $\lceil\gamma\rceil$ denotes the closest integer number greater or equal the real number $\gamma$. To verify formula (3) we consider two cases. 
First case: $l+1$ is collapsing. This means, that $j(l+1, k, 1) \leq k-2$, which is equivalent to $l+1 \leq k-1$. Hence, $l+\left\lceil\frac{l+1}{k-1}\right\rceil=l+1$, as claimed.

Second case: $l+1$ is not collapsing. In this case, $j(l+1, k, 1)=k-1$. Thus, the first collapsing number is the smallest integer $c_{1}$ with the property, that $k-1+k\left(c_{1}-(l+1)\right) \geq c_{1}$. Solving for $c_{1}$ yields $c_{1}=\left\lceil\frac{k(l+1)}{k-1}\right\rceil-1=l+\left\lceil\frac{l+1}{k-1}\right\rceil$. This establishes (3).

Now let $c_{m}$ denote the $m$ th collapsing number and $d_{m}:=j\left(c_{m}, k, c_{m}-l\right)$. For $d_{1}$ we obtain the formula

$$
d_{1}=\left(\left\lceil\frac{l+1}{k-1}\right\rceil \cdot k-1\right) \bmod \left(l+\left\lceil\frac{l+1}{k-1}\right\rceil\right) .
$$

To see this, we consider again the following two cases:

First case: $l+1$ is a collapsing number. In this case $d_{1}=j(l+1, k, 1)=$ $(k-1) \bmod (l+1)=\left(\left\lceil\frac{l+1}{k-1}\right\rceil k-1\right) \bmod \left(l+\left\lceil\frac{l+1}{k-1}\right\rceil\right)$, since $k-1 \geq l+1$.

Second case: $l+1$ is not a collapsing number. In this case $k-1<l+1$, and $d_{1}=k-1+k\left(c_{1}-(l+1)\right)-c_{1}=k-1+k\left(c_{1}-(l+1)\right) \bmod c_{1}=$ $\left(\left\lceil\frac{l+1}{k-1}\right\rceil \cdot k-1\right) \bmod \left(l+\left\lceil\frac{l+1}{k-1}\right\rceil\right)$ by definition of $c_{1}$ and by $(3)$.

If $c_{m} \leq n<c_{m+1}$ then by the recursive formula of Theorem 1 we obtain

$$
j(n, k, n-l)=\left(n-c_{m}\right) k+d_{m} .
$$

Hence, to compute $j(n, k, n-l)$ it is sufficient to know the sequences $c_{m}$ and $d_{m}$.

Let us start with a recursive formula for $c_{m}$ and $d_{m}$ (for fixed $k$ and $l$ ).

If $c_{m}$ and $d_{m}$ are known for some $m$, then $c_{m+1}=c_{m}+s$ where $s$ is the least integer such that $s \cdot k+d_{m} \geq c_{m}+s$ and $d_{m+1}=s(k-1)+d_{m}-c_{m}$. Hence we obtain

$$
s=\left\lceil\frac{c_{m}-d_{m}}{k-1}\right\rceil
$$

and thus

$$
c_{m+1}=\left\lceil\frac{k c_{m}-d_{m}}{k-1}\right\rceil
$$

and after a short calculation

$$
d_{m+1}=\left(d_{m}-c_{m}\right) \bmod (k-1) .
$$


It will be convenient to write the formula for $c_{m+1}$ in the form

$$
c_{m+1}=\left\lceil\frac{k c_{m}-d_{m}}{k-1}\right\rceil=: \frac{k c_{m}+\sigma_{m}}{k-1}
$$

with $\sigma_{m} \in\{-(k-2), \ldots, k-3, k-2\}$. Rewriting the definition (8) for $\sigma_{m}$ we obtain

$$
\sigma_{m}=\left(d_{m}-c_{m}\right) \bmod (k-1)-d_{m}=d_{m+1}-d_{m}
$$

The following table which we need later gives the values of $d_{m+1}$ and $\sigma_{m}$ if

\begin{tabular}{|c|c|c|c|}
\hline$c_{m} \bmod (k-1)$ & $d_{m}$ & $\sigma_{m}$ & $d_{m+1}$ \\
\hline 0 & 0 & 0 & 0 \\
\hline 0 & 1 & 0 & 1 \\
\hline 0 & 2 & 0 & 2 \\
\hline$\vdots$ & $\vdots$ & $\vdots$ & $\vdots$ \\
\hline 0 & $\mathrm{k}-2$ & 0 & $\mathrm{k}-2$ \\
\hline 1 & 0 & $\mathrm{k}-2$ & $\mathrm{k}-2$ \\
\hline 1 & 1 & -1 & 0 \\
\hline 1 & 2 & -1 & 1 \\
\hline$\vdots$ & $\vdots$ & $\vdots$ & $\vdots$ \\
\hline 1 & $\mathrm{k}-2$ & -1 & $\mathrm{k}-3$ \\
\hline 2 & 0 & $\mathrm{k}-3$ & $\mathrm{k}-3$ \\
\hline 2 & 1 & $\mathrm{k}-3$ & $\mathrm{k}-2$ \\
\hline 2 & 2 & -2 & 0 \\
\hline$\vdots$ & $\vdots$ & $\vdots$ & $\vdots$ \\
\hline 2 & $\mathrm{k}-2$ & -2 & $\mathrm{k}-4$ \\
\hline$\vdots$ & $\vdots$ & $\vdots$ & $\vdots$ \\
\hline $\mathrm{k}-2$ & 0 & +1 & 1 \\
\hline $\mathrm{k}-2$ & 1 & +1 & 2 \\
\hline$\vdots$ & $\vdots$ & $\vdots$ & $\vdots$ \\
\hline $\mathrm{k}-2$ & $\mathrm{k}-3$ & +1 & $\mathrm{k}-2$ \\
\hline $\mathrm{k}-2$ & $\mathrm{k}-2$ & $-\mathrm{k}+2$ & 0 \\
\hline
\end{tabular}
$c_{m} \bmod (k-1)$ and $d_{m}$ are known.

Table 1 
Summary: Starting with (3) and (4) we can recursively compute the sequences $c_{m}, d_{m}$ and $\sigma_{m}$ (using either Table 1 or the formulas (6)-(9)). Then $j(n, k, n-l)$ is given by (5) .

\section{How does the sequence $c_{m}$ grow?}

Also for this section let $k>1$ and $l$ be fixed. If $\gamma$ is a real number, then $\operatorname{round}(\gamma):=\gamma+\epsilon$ where $-\frac{1}{2}<\epsilon \leq \frac{1}{2}$ is such that $\gamma+\epsilon$ is an integer number. Let us start with the following technical lemma:

LEMMA 1. Let $\sigma_{0}, \sigma_{1}, \ldots$ be a sequence of real numbers and for $i=0,1, \ldots$ let $p_{i}(x):=\sum_{j=0}^{i} \sigma_{j} x^{j}$. Then the sets

$$
\begin{aligned}
& M^{+}:=\left\{(x, y): y \geq \max \left\{p_{0}(x), p_{1}(x), \ldots, p_{m}(x)\right\}\right\}, \\
& M^{-}:=\left\{(x, y): y \leq \min \left\{p_{0}(x), p_{1}(x), \ldots, p_{m}(x)\right\}\right\}
\end{aligned}
$$

are convex for any $m$.

Proof: The proof is by induction: For $m=0$ the assertion is trivial. Now suppose we have verified the convexity of the assigned sets for $m<m_{0}$ and suppose that convexity (say for $M^{+}$) fails to be true for the index $m_{0}$ and a sequence $\left\{\sigma_{j}\right\}$. We may assume that $\sigma_{0}=0$ and that there exists an $x_{0}>0$ with $p_{i}^{\prime \prime}\left(x_{0}\right)<0$ and $p_{i}^{\prime}\left(x_{0}\right)>p_{i}\left(x_{0}\right) / x_{0}$ where $\left(x_{0}, p_{i}\left(x_{0}\right)\right) \in \partial M^{+}$. By choosing $\sigma_{1}$ appropriately we may further assume that $p_{i}^{\prime}\left(x_{0}\right)>0$. Now consider the family $q_{j}(x):=p_{j}(x) / x\left(j=1, \ldots m_{0}\right)$ of polynomials. For $x_{0}$ there holds $q_{i}^{\prime \prime}\left(x_{0}\right)=p_{i}^{\prime \prime}\left(x_{0}\right) / x_{0}-2 p_{i}^{\prime}\left(x_{0}\right) / x_{0}^{2}+2 p_{i}\left(x_{0}\right) / x_{0}^{3}<p_{i}^{\prime \prime}\left(x_{0}\right) / x_{0}<0$ which contradicts the convexity of the sets $M^{+}$for the index $m_{0}-1$.

ThEOREM 2. The limit $\alpha:=\lim _{m \rightarrow \infty} c_{m}\left(1-\frac{1}{k}\right)^{m}$ (depending on $k$ and $l$ ) exists and for all $m \in \mathbb{N}$ there holds

$$
\left|c_{m}-\alpha \cdot\left(\frac{k}{k-1}\right)^{m}\right|<1-\frac{2}{k}
$$

for $k>2$ and $c_{m}=\alpha \cdot 2^{m}$ for $k=2$. Hence, for $k \in\{2,3,4\}$ there holds

$$
c_{m}=\operatorname{round}\left(\alpha \cdot\left(\frac{k}{k-1}\right)^{m}\right)
$$

and for $k \geq 5$

$$
\mid c_{m}-\text { round }\left(\alpha \cdot\left(\frac{k}{k-1}\right)^{m}\right) \mid \leq 1 \text {. }
$$


Proof: 1. Step: By induction we get from (8) that

$$
\begin{aligned}
c_{m+1} & =\left(\frac{k}{k-1}\right)^{m} \cdot c_{1}+\frac{1}{k-1}\left(\left(\frac{k}{k-1}\right)^{m-1} \cdot \sigma_{1}+\left(\frac{k}{k-1}\right)^{m-2} \cdot \sigma_{2}+\ldots+\sigma_{m}\right) \\
& =\left(\frac{k}{k-1}\right)^{m} \cdot c_{1}+\frac{1}{k-1} \sum_{i=1}^{m} \sigma_{i}\left(\frac{k}{k-1}\right)^{m-i} .
\end{aligned}
$$

Hence there holds

$$
c_{m+1}\left(1-\frac{1}{k}\right)^{m+1}=c_{1}\left(1-\frac{1}{k}\right)+\sum_{i=1}^{m} \frac{\sigma_{i}}{k-1}\left(1-\frac{1}{k}\right)^{i+1}
$$

Now

$$
f(z):=c_{1} z+\sum_{i=1}^{\infty} \frac{\sigma_{i}}{k-1} z^{i+1}
$$

defines an analytic function and the convergence radius of the power series is $\geq 1$. Thus, we get

$$
\alpha=\lim _{m \rightarrow \infty} c_{m}\left(1-\frac{1}{k}\right)^{m}=f\left(1-\frac{1}{k}\right) .
$$

2. Step: For fixed $h \in \mathbb{N}$ we define $\alpha_{h}:=c_{h}\left(1-\frac{1}{k}\right)^{h}$ and for $m \in$ $\{1,2, \ldots, h\} e_{m}:=\alpha_{h}\left(\frac{k}{k-1}\right)^{m}$, i.e. $e_{h}=c_{h}$. Now we will show that $e_{m}$ is a good approximation for $c_{m}$ for $1 \leq m \leq h$. To simplify the notation let $q_{k}:=\frac{k}{k-1}$.

$$
\text { (14) } \begin{aligned}
& e_{m+1}-c_{m+1}= \\
= & \frac{c_{h}}{q_{k}^{h}} \cdot q_{k}^{m+1}-q_{k}^{m} \cdot c_{1}-\frac{1}{k-1} \sum_{i=1}^{m} \sigma_{i} q_{k}^{m-i} \\
= & q_{k}^{m+1-h} \cdot\left(q_{k}^{h-1} \cdot c_{1}+\frac{1}{k-1} \sum_{i=1}^{h-1} \sigma_{i} q_{k}^{h-1-i}\right)-q_{k}^{m} \cdot c_{1}-\frac{1}{k-1} \sum_{i=1}^{m} \sigma_{i} q_{k}^{m-i} \\
= & q_{k}^{m+1-h} \cdot \frac{1}{k-1} \sum_{i=1}^{h-1} \sigma_{i} q_{k}^{h-1-i}-\frac{1}{k-1} \sum_{i=1}^{m} \sigma_{i} q_{k}^{m-i} \\
= & \frac{1}{k-1} \sum_{i=1}^{h-1} \sigma_{i} q_{k}^{m-i}-\frac{1}{k-1} \sum_{i=1}^{m} \sigma_{i} q_{k}^{m-i} \\
= & \frac{1}{k-1} \sum_{i=m+1}^{h-1} \sigma_{i} q_{k}^{m-i} \\
= & \frac{1}{k} \sum_{j=0}^{h-m-2} \sigma_{j+m+1}\left(\frac{k-1}{k}\right)^{j} .
\end{aligned}
$$


Note that by (9) there holds

$$
\left|\sum_{j=j_{0}}^{j_{1}} \sigma_{j}\right|=\left|d_{j_{1}+1}-d_{j_{0}}\right| \leq k-2
$$

for arbitrary $j_{0}, j_{1}$. Consider the polynomials $p_{i}(x)=\sum_{j=0}^{i} \sigma_{j+m+1} x^{j}$. We have $p_{i}(0)=\sigma_{m+1} \in\{-k+2, \ldots, k-2\}$ and $p_{i}(1)=\sum_{j=0}^{i} \sigma_{j+m+1} \in\{-k+$ $2, \ldots, k-2\}$. Hence by Lemma 1 we obtain that $\left|p_{i}\left(1-\frac{1}{k}\right)\right| \in[-k+2, k-2]$ and hence that the right hand side of (14) has a value in $\left[-1+\frac{2}{k}, 1-\frac{2}{k}\right]$. Thus we have

$$
\left|e_{m+1}-c_{m+1}\right|=\left|\alpha_{h}\left(\frac{k}{k-1}\right)^{m+1}-c_{m+1}\right| \leq 1-\frac{2}{k} .
$$

The first step allows to pass to the limit $h \rightarrow \infty$ in (16) and we obtain

$$
\left|c_{m}-\alpha \cdot\left(\frac{k}{k-1}\right)^{m}\right| \leq 1-\frac{2}{k} \text {. }
$$

Now suppose that for some $m$ there holds

$$
\begin{aligned}
1-\frac{2}{k} & =\left|c_{m+1}-\alpha\left(\frac{k}{k-1}\right)^{m+1}\right|= \\
& =\lim _{h \rightarrow \infty}\left|c_{m+1}-\alpha_{h}\left(\frac{k}{k-1}\right)^{m+1}\right|= \\
& =\lim _{h \rightarrow \infty}\left|\frac{1}{k} \sum_{j=0}^{h-m-2} \sigma_{j+m+1}\left(1-\frac{1}{k}\right)^{j}\right|,
\end{aligned}
$$

and hence

$$
k-2=\lim _{h \rightarrow \infty} p_{h}\left(1-\frac{1}{k}\right)
$$

(by changing sign if necessary). Observe that

$$
\begin{aligned}
& p_{h}(0)=\sigma_{m+1} \in\{-k+2,-k+3, \ldots, k-2\}, \\
& p_{i}(1)=\sum_{r=0}^{i} \sigma_{r+m+1} \in\{-k+2,-k+3, \ldots, k-2\} .
\end{aligned}
$$

Hence from Lemma 1 it follows that $\sigma_{m+1}=k-2$. Furthermore we claim that $\sigma_{j}=0$ for $j>m+1$. In fact, if $\sigma_{j}$ is the first non-zero coefficient after $\sigma_{m+1}$, then $\sigma_{j}<0$ by (19). We replace $\sigma_{j}$ by $\tilde{\sigma}_{j}=\sigma_{j}+1$ and $\sigma_{j+1}$ by $\tilde{\sigma}_{j+1}=\sigma_{j+1}-1$ without violating (18) and (19) if $k>2$. We denote the modified polynomials by $\tilde{p}_{h}$. It follows that $\lim _{h \rightarrow \infty} \tilde{p}_{h}\left(1-\frac{1}{k}\right)>k-2$ and hence $\tilde{p}_{h}\left(1-\frac{1}{k}\right)>k-2$ for some $h$ large enough. But this contradicts Lemma 1 and $\sigma_{j}=0$ for $j>m+1$ is established. This implies that for all 
$j \in \mathbb{N}$ there holds $c_{m+1+j}=\left(\frac{k}{k-1}\right)^{j} c_{m+1} \in \mathbb{N}$ which is clearly impossible if $k>2$. Thus we have (10) and (11)-(12) follow immediately.

Remarks: (a) Note that if the sequence $\left\{\sigma_{m}\right\}$ (or equivalently the sequence $\left\{d_{m}\right\}$ ) would be periodic, then $\alpha$ would be a rational number.

(b) As we can see from the second step in the above proof we can use the approximation $\alpha_{h}:=c_{h}\left(1-\frac{1}{k}\right)^{h}$ for $\alpha$ and that then the assertions of Theorem 2 hold with $\alpha_{h}$ in place of $\alpha$ at least for indices $m \leq h$.

As a further fact we state the following

Lemma 2. For $k=5$ the following is true: Let $l$ be fixed and let $c_{m}$ be a collapsing number which is a multiple of 4 , then there holds

$$
c_{m}=\operatorname{round}\left(\alpha \cdot\left(\frac{5}{4}\right)^{m}\right) \text {. }
$$

Proof: Essentially we repeat the proof of the previous theorem: Notice that $\frac{c_{m}}{4} \in \mathbb{N}$ implies $\sigma_{m}=0$ by Table 1 . This allows to obtain a better estimate in (14): In fact Lemma 1 implies now that $\left|p_{i}\left(1-\frac{1}{5}\right)\right| \in\left[-\frac{12}{5}, \frac{12}{5}\right]$ and hence the right hand side of $(14)$ has a value in $\left[-\frac{12}{25}, \frac{12}{25}\right]$.

\section{Some special cases}

In this section we consider the cases when $k$ equals 2,3 or 4 and the case when $k \geq 5$. The case when $k$ equals 2 is very easy and we will give different explicit (in $n$ and $i$ ) formulas to calculate $j(n, 2, i)$. For $k$ equals 3 , we will give an explicit (in $n$ ) formula to calculate $j(n, 3, i)$. If $k$ equals 4 , although we can calculate the collapsing numbers $c_{m}$ precisely, the corresponding explicit formula gives only an approximation $\tilde{\jmath}(n, 4, i)$ having the property that $j(n, 4, i) \in\{\tilde{\jmath}(n, 4, i), \tilde{\jmath}(n, 4, i)+1\}$. In general for $k \geq 5$ we obtain $j(n, k, i) \in\{\tilde{\jmath}(n, k, i), \ldots, \tilde{\jmath}(n, k, i)+2 k-2\}$.

The case when $k$ equals 2:

If $k=2$ then equation (11) states that

$$
c_{m}=\operatorname{round}\left(\alpha \cdot 2^{m}\right)
$$

and because $c_{1}=l+(l+1)=2 l+1$ we get $2 l+1=2 \alpha$, hence $\alpha=\frac{2 l+1}{2}$ and

$$
c_{m}=(2 l+1) \cdot 2^{m-1} .
$$


Because $\sigma_{m}=d_{m}=0$ for all $m, j(n, 2, n-l)=2 \cdot\left(n-c_{m}\right)$ where $c_{m} \leq$ $n<c_{m+1}$. So, by (21), $m-1=\left\lfloor\log _{2}\left(\frac{n}{2 l+1}\right)\right\rfloor$ and finally

$$
j(n, 2, n-l)=2\left(n-(2 l+1) \cdot 2^{\left.\log _{2} n-\log _{2}(2 l+1)\right\rfloor}\right) .
$$

If $l=0$, then by $(22), j(n, 2, n)=2\left(n-2^{\left\lfloor\log _{2} n\right\rfloor}\right)$. Thus we get the binary code of $j(n, 2, n)$ if we first write $n$ in the binary code, cancel the leading " 1 " (note that this is $n-\left\lfloor\log _{2} n\right\rfloor$ ) and then join to this binary number a " 0 " at the end. (This interpretation of the formula $2\left(n-2^{\left.\log _{2} n\right\rfloor}\right)$ can be found in [1]).

Before we continue with larger values of $k$, we need the following lemma:

Lemma 3. For $k \geq 3$ there holds with $e_{m}=\alpha\left(\frac{k}{k-1}\right)^{m}$ (and $\alpha$ as in Theorem 1)

(i) $e_{m}-c_{m}<0 \Longrightarrow d_{m} \neq 0$

(ii) $e_{m}-c_{m}>0 \Longrightarrow d_{m} \neq k-2$.

Proof: From Section 4 we infer

$$
\begin{aligned}
e_{m}-c_{m} & =\frac{1}{k} \sum_{j=0}^{\infty} \sigma_{j+m}\left(1-\frac{1}{k}\right)^{j}= \\
& =\frac{1}{k} \sum_{j=0}^{\infty}\left(d_{m+j+1}-d_{m+j}\right)\left(1-\frac{1}{k}\right)^{j} .
\end{aligned}
$$

(i) Suppose $d_{m}=0$. Applying Lemma 1 as in the proof of Theorem 2 we immediately conclude $e_{m}-c_{m} \geq 0$.

(ii) Analogue to $(i)$.

The case when $k$ equals 3 :

If $k=3$, then equation (11) states that

$$
c_{m}=\operatorname{round}\left(e_{m}\right)
$$

with $e_{m}=\alpha \cdot\left(\frac{3}{2}\right)^{m}$. To compute $j(n, 3, n-l)$ by (5) it is sufficient to know $c_{m}$ satisfying $c_{m} \leq n<c_{m+1}$ and the corresponding $d_{m}$. To find $c_{m}$ is easy: For $n \geq 5$ the appropriate $m$ is either $\tilde{m}-1$ or $\tilde{m}$ where

$$
\tilde{m}=\operatorname{round}\left(\frac{\log \frac{n}{\alpha}}{\log \frac{3}{2}}\right)
$$

(use (23) to decide which one is the right choice). 
Now according to Lemma 3 we find $d_{m} \in\{0,1\}$ as follows:

$$
\begin{aligned}
& \text { If } e_{m}-c_{m}<0 \text { then } d_{m}=1 \\
& \text { If } e_{m}-c_{m}>0 \text { then } d_{m}=0 .
\end{aligned}
$$

Finally $j(n, 3, n-l)$ is given by (5).

Example: Let us use the above method in the original Josephus problem as described in the introduction, i.e. we want to calculate $j(41,3,41)$. The value of $\alpha$ for $k=3$ and $l=0$ is $\alpha=0.8111 \ldots$ (see Theorem 2).

1. Step: From (24) we find $\tilde{m}=10$ and by (23) $c_{\tilde{m}}=47>41$. Thus $m=9$ and $c_{9}=31$ (again by (23)).

2. Step: $e_{9}-c_{9}=0.1827 \ldots>0$. Hence $d_{9}=0$.

3. Step: $j(41,3,41)=3 \cdot\left(41-c_{9}\right)+d_{9}=30$ (i.e. position 31 if we start numbering at 1 rather than at 0$)$. This was in fact Josephus' place!

The case when $k$ equals 4 :

For $k=4$ equation (11) states that

$$
c_{m}=\operatorname{round}\left(e_{m}\right)
$$

with $e_{m}=\alpha \cdot\left(\frac{4}{3}\right)^{m}$. To compute $j(n, 4, n-l)$ by (5) we would again need to know $c_{m}$ satisfying $c_{m} \leq n<c_{m+1}$ and the corresponding $d_{m}$. We find $c_{m}$ as above for $k=3$ : For $n \geq 7$ the appropriate $m$ is either $\tilde{m}-1$ or $\tilde{m}$ where

$$
\tilde{m}=\text { round }\left(\frac{\log \frac{n}{\alpha}}{\log \frac{4}{3}}\right)
$$

(use (25) to decide which one is the right choice).

Unfortunately there seems to be no (easy) way to compute $d_{m} \in\{0,1,2\}$. However according to Lemma 3 we find that:

$$
\begin{aligned}
& \text { If } e_{m}-c_{m}<0 \text { then } d_{m}=1 \text { or } 2 \\
& \text { If } e_{m}-c_{m}>0 \text { then } d_{m}=0 \text { or } 1 .
\end{aligned}
$$

Now, if we choose $\tilde{d}_{m}=1$ in the first case and $\tilde{d}_{m}=0$ in the second case and define $\tilde{j}(n, 4, n-l)=4\left(n-c_{m}\right)+\tilde{d}_{m}$ then we have by (5) that $j(n, 4, n-l) \in\{\tilde{\jmath}(n, 4, n-l), \tilde{\jmath}(n, 4, n-l)+1\}$.

We want to emphasize that in many cases we still can decide which possibility is the right one. For this purpose we give several lemmas: 
Lemma 4. For $\sigma_{m}=3 c_{m+1}-4 c_{m}$ there holds

(i) If $\sigma_{m}=2$ then $d_{m}=0$.

(ii) If $\sigma_{m}=-2$ then $d_{m}=2$.

(iii) If $\sigma_{m}=1$ then $d_{m} \neq 2$.

(iv) If $\sigma_{m}=-1$ then $d_{m} \neq 0$.

Proof: The formula for $\sigma_{m}$ follows just from definition (8). Then (i)-(iv) follow from Table 1 .

Lemma 5. For all $l$ there is a number $n_{l} \geq k$, such that for all $n \geq n_{l}$ the following is true:

$$
s=n \bmod k \quad \Longrightarrow \quad j(n, k, n-l) \neq k-1-s .
$$

Proof: If $s=0$, then of course $(k-1) \bmod n$ is the first number which is removed.

If $s>0$, then the first number belonging to the set $\{n-1,0,1, \ldots, k-2\}$ which is removed is $k-1-s$.

For both cases if we choose $n_{l}$ large enough, the number $k-1-s$ is removed too early.

As an immediate consequence of this lemma we get:

COROLlaRY. For fixed $l$ and $k$ there is an $m_{0}$, such that for all $m \geq m_{0}$ the following is true:

$$
s=c_{m} \bmod k \quad \Longrightarrow \quad d_{m} \neq k-1-s .
$$

Further we have

LemmA 6. For all $l$ there is a number $n_{l} \geq k$, such that for all $n \geq n_{l}$ the following is true:

$$
1=n \bmod k^{2} \quad \Longrightarrow \quad j(n, k, n-l) \neq k-3 .
$$

Proof: Like the proof of Lemma 5.

The case when $k \geq 5$ :

Here we can no longer compute the sequence $\left\{c_{j}\right\}$ exactly. But according to Theorem 2 we still have that $c_{m} \in\left\{\left\lfloor\alpha\left(\frac{k}{k-1}\right)^{m}\right\rfloor,\left\lceil\alpha\left(\frac{k}{k-1}\right)^{m}\right\rceil\right\}$. It is now hard to say anything about the $d_{m}$ but if we define $\tilde{c}_{m}:=\left\lfloor\alpha\left(\frac{k}{k-1}\right)^{m}\right\rfloor, \tilde{d}_{m}:=0$ 
and $\tilde{\jmath}(n, k, n-l):=k\left(n-\tilde{c}_{m}\right)+\tilde{d}_{m}$ then we have that $j(n, k, n-l) \in$ $\{\tilde{\jmath}(n, k, n-l), \ldots, \tilde{\jmath}(n, k, n-l)+2 k-2\}$ (the discussion of the case $n=\tilde{c}_{m}$ is left to the reader). Thus if $n>2 k-2$ we can say at least which is not the $i$ th position to be removed.

\section{An algorithm to compute $j(n, k, i)$ for $k \geq 4$}

The problem in the previous section was to compute (for given $n$ and $k \geq 4$ ) the corresponding $c_{m}$ with $c_{m} \leq n<c_{m+1}$ and the value of $d_{m}$. Let us define $c_{m}^{1}:=\left\lfloor\alpha\left(\frac{k}{k-1}\right)^{m}\right\rfloor$ and $c_{m}^{2}:=\left\lceil\alpha\left(\frac{k}{k-1}\right)^{m}\right\rceil$. We denote $d_{m}^{j}:=j$. Let $c_{m+h}^{i j}$ be the number that is obtained by iterating (6) and (7) $h$-times starting with $c_{m}^{i}$ and $d_{m}^{j}$. The idea is now that $\left|c_{m+h}^{i j}-c_{m+h}\right|$ grows exponentially with $h$ if $\left(c_{m}^{i}, d_{m}^{j}\right) \neq\left(c_{m}, d_{m}\right)$. To be more precise, let us define the matrix

$$
A_{h}^{i j}:=\left|c_{m+h}^{i j}-\alpha\left(\frac{k}{k-1}\right)^{m+h}\right|
$$

for $i \in\{1,2\}$ and $j \in\{0,1, \ldots, k-2\}$. Starting with $h=0$ we keep iterating this matrix until there is only one entry left which satisfies $A_{h}^{\sigma \rho}<1$. Then we may conclude that

$$
c_{m}=c_{m}^{\sigma}, \quad d_{m}=d_{m}^{\rho} .
$$

Then again by (5) we may compute $j(n, k, i)$.

Now we give a rough estimate for the number $h$ of iterations of the matrix $A$ which need to be carried out in order to decide $c_{m}$ and $d_{m}$ : To do this we assume that the sequence $\left\{d_{j}\right\}$ is pseudo-random, i.e. we assume the following hypothesis:

Hypothesis. Let $\delta_{i, j}$ be 1 if $i=j$ and 0 otherwise. Then for all $d \in$ $\{0,1, \ldots, k-2\}$ :

$$
\lim _{m \rightarrow \infty}\left(\frac{1}{m} \cdot \sum_{r=1}^{m} \delta_{d, d_{r}}\right)=\frac{1}{k-1} .
$$

We assume that $c_{m}^{i}=c_{m}$ and $d_{m}^{j}=d_{m}-1$ (the other cases are similar). So if we compute the Josephus number $\tilde{\jmath}(n, k, n-l)\left(n \geq c_{m}\right)$ with $d_{m}^{j}$ and compare with the true $j(n, k, n-l)$, we see that the difference is 1 . With (1) we see that if the first time $d_{m+r}=0$ occurs then the difference of $\tilde{\jmath}(n, k, n-l)$ and $j(n, k, n-l)\left(n>c_{m+r}\right)$ gets 2 , and so on. Now if the difference has grown to a value larger than $2 k$, we may conclude that $\left|c_{m+h}-c_{m+h}^{i j}\right| \geq 2$ and hence $\left|\alpha\left(\frac{k}{k-1}\right)^{m+h}-c_{m+h}^{i j}\right| \geq 1$. Using the hypothesis we easily compute that the expected value for $h$ is estimated by $E[h] \leq k^{2}$. 
A refined analysis gives a better bound for the expected value of $h$, namely $E[h] \leq k(2+\log k)$. (Notice that similar results hold under much weaker assumptions than the Hypothesis)

Example: Let us calculate $j(n, k, n-l)$ for $k=7, l=2001$ and the prime number

$$
n=11111111111111111111111 .
$$

By Theorem 2 we find $\alpha=2001.41696981983172 \ldots$ and then $\tilde{m}=\operatorname{round}\left(\frac{\log \frac{n}{\alpha}}{\log \frac{k}{k-1}}\right)=280$. With $e_{i}=\alpha\left(\frac{k}{k-1}\right)^{i}$ we find $\operatorname{round}\left(e_{280}\right)=$ $11128552382382930685534>n$ and hence we have to choose $m=\tilde{m}-1=$ 279 such that $\left\lceil e_{279}\right\rceil \leq n<\left\lfloor e_{280}\right\rfloor$. After iterating the matrix $A_{h}^{i j} 19$ times we find that

$$
A_{19}^{i j}=\left(\begin{array}{cccccc}
9.92 & 11.92 & 12.92 & 18.92 & 20.92 & 22.92 \\
10.07 & 5.07 & 5.07 & 1.07 & 0.07 & 2.92
\end{array}\right) .
$$

Thus $c_{279}=\left\lceil e_{279}\right\rceil=9538759184899654873315$ and $d_{279}=4$. Finally we obtain

$$
\begin{aligned}
& j(11111111111111111111111,7,11111111111111111109110)= \\
& \quad=7 \cdot(11111111111111111111111-9538759184899654873315)+4= \\
& =11006463483480193664576 . \square
\end{aligned}
$$

\section{REFERENCES}

[1] Burde, K.: "Das Problem der Abzählreime und Zahlenentwicklungen mit gebrochenen Basen". J. of Number Theory 26 (1987), 192-209

[2] Jakóbczyk, F.: "On the generalized Josephus problem". Glasgow Math. J. 14 (1973), 168-173

[3] Josephus, F.: "The jewish war, Book III". Translated by H. S. Thackeray, Heinemann (1927), 341-366, 387-391

[4] Nörlund, N. E.: "Vorlesungen über Differenzenrechnung", Grundlehren d. math. Wissensch. 13, Springer, Berlin 1924

[5] Robinson, W. J.: "The Josephus problem". Math. Gazette 44 (1960), 47-52

[6] Rouse Ball, W. W.: "Mathematical recreations and essays". Reprint New York (1962), 32-36

[7] Woodhouse, D.: "The extended Josephus problem". Rev. Mat. Hisp.-Amer. (4) 33 (1973), 207-218 
Lorenz HaLbEISEN Norbert HUNGERBÜHLER

Mathematik Departement Univerität Freiburg

ETH Zentrum HG G53 Rheinstrasse 10

CH - 8092 Zürich

Switzerland

D - 79100 Freiburg

halbeis@math.ethz.ch

Germany

buhler@math.ethz.ch 